Supporting Information for

\title{
A Nonaqueous MEA/PEG200 Absorbent with High Efficiency and Low Energy Consumption for $\mathrm{CO}_{2}$ Capture
}

Wen Tian ${ }^{1}$, Kui Ma ${ }^{1}$, Junyi Ji ${ }^{1}$, Siyang Tang ${ }^{1}$, Shan Zhong ${ }^{1}$, Changjun Liu ${ }^{1,2}$, Hairong Yue $^{1,2}$, and Bin Liang ${ }^{1,2 *}$

${ }^{1}$ Low-Carbon Technology and Chemical Reaction Engineering Laboratory, School of Chemical Engineering, Sichuan University, Chengdu 610065, China;

${ }^{2}$ Institute of New Energy and Low-Carbon Technology, Sichuan University, Chengdu 610207, China

*Corresponding author: $\underline{\text { liangbin@scu.edu.cn }}$ 


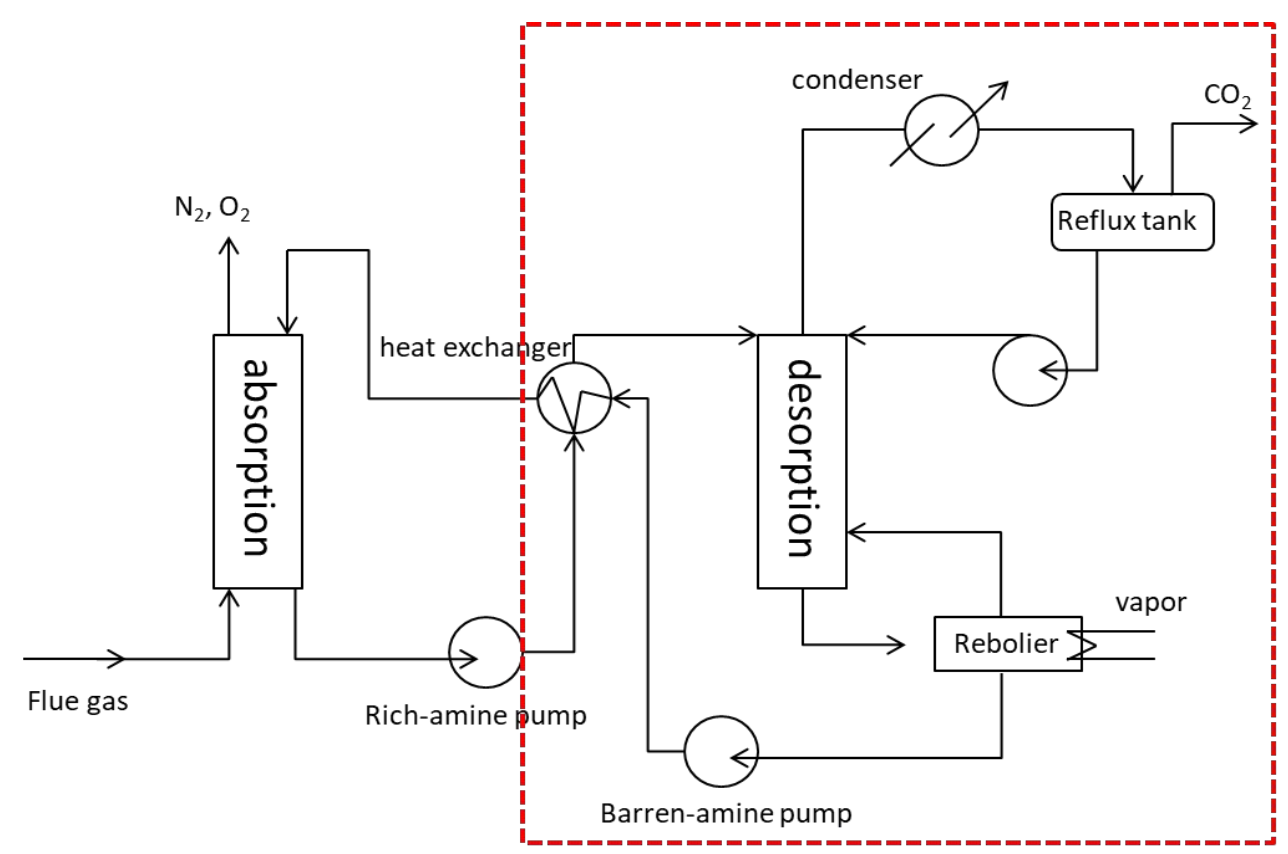

Figure S1. Flow chart of $\mathrm{CO}_{2}$ capture in flue gas by chemical absorption. 


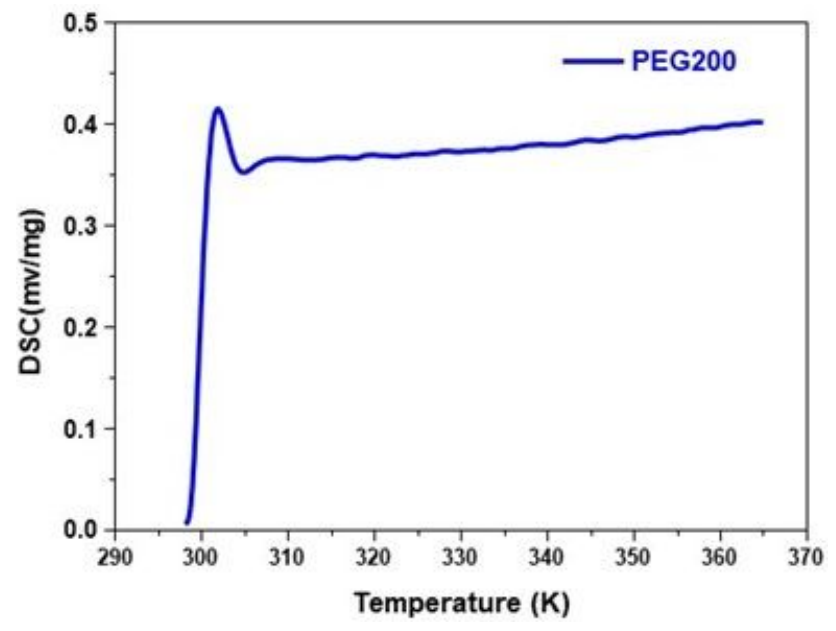

Figure S2. The DSC curve of the PEG200 solvent. 


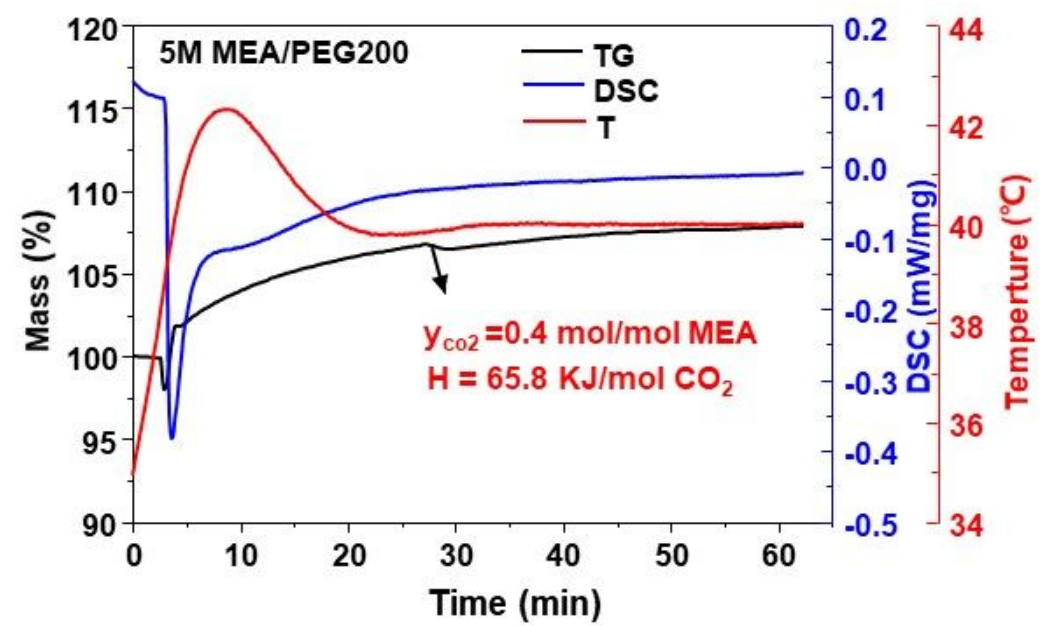

Figure S3. TG-DSC diagram of $\mathrm{CO}_{2}$ absorption process of $5 \mathrm{M}$ MEA/PEG200. 


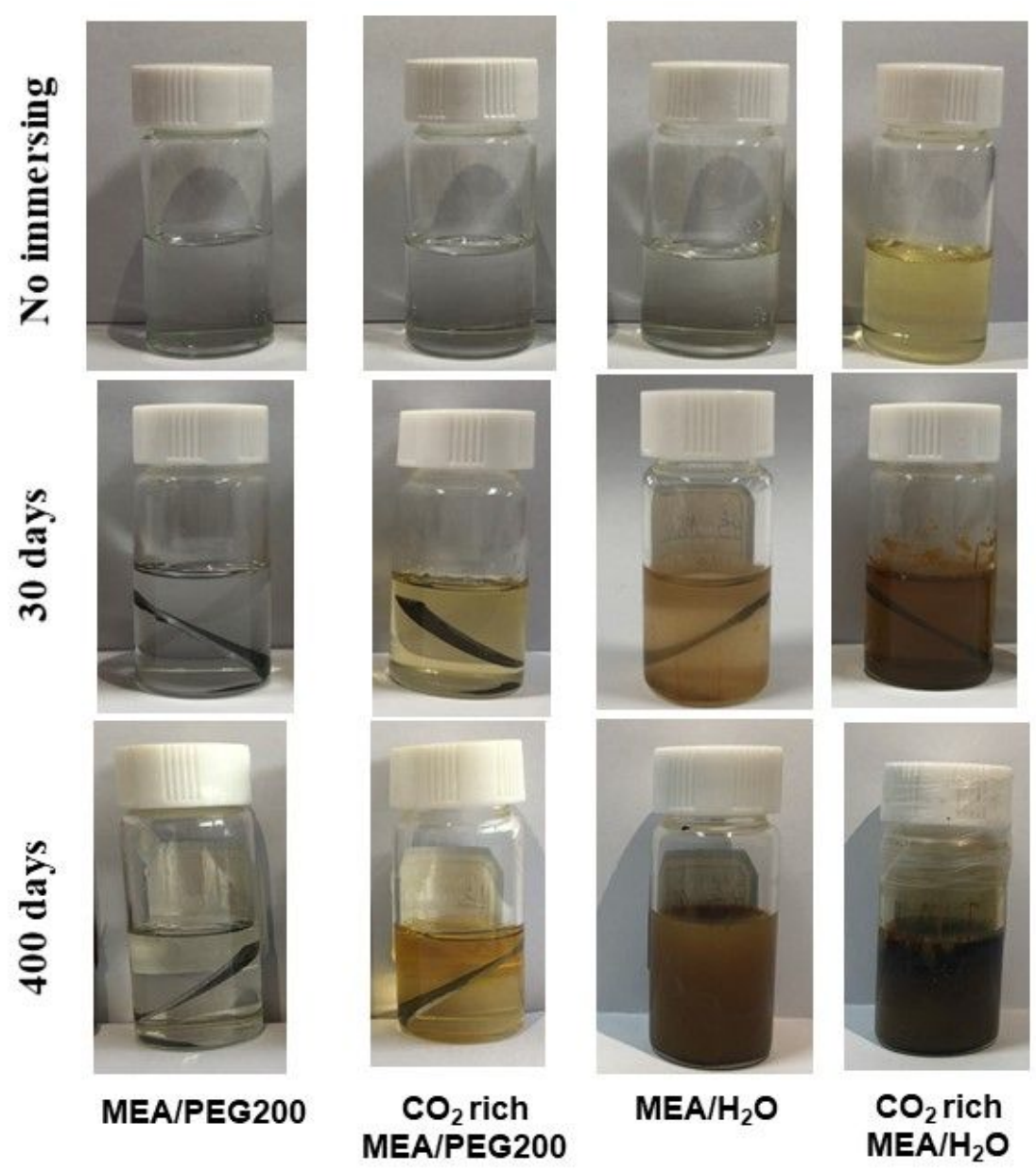

Figure S4. Evolution of the fresh and $\mathrm{CO}_{2}$ rich solution before and after immersion of carbon steel plate for 30 to 400 days. 

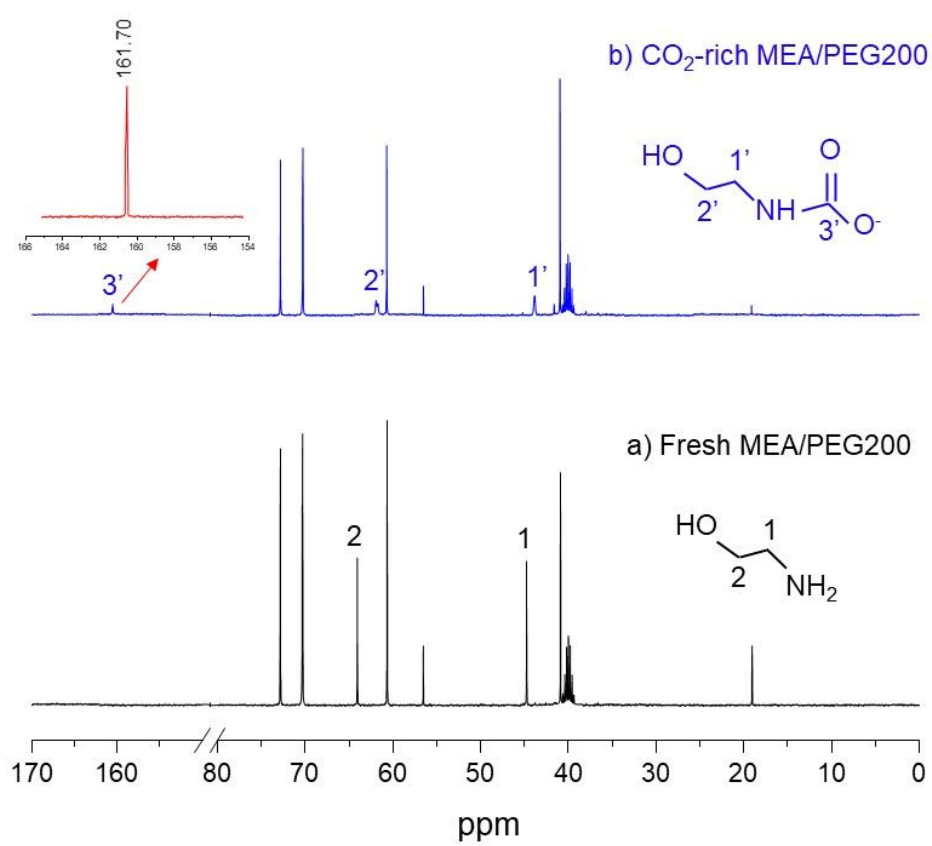

Figure S5. ${ }^{13} \mathrm{C}$ NMR spectra of MEA/PEG200 absorbents in DMSO-d $\mathrm{d}_{6}$. (a) Fresh MEA/PEG200 absorbent; (b) $\mathrm{CO}_{2}$-rich MEA/PEG200 absorbent. 


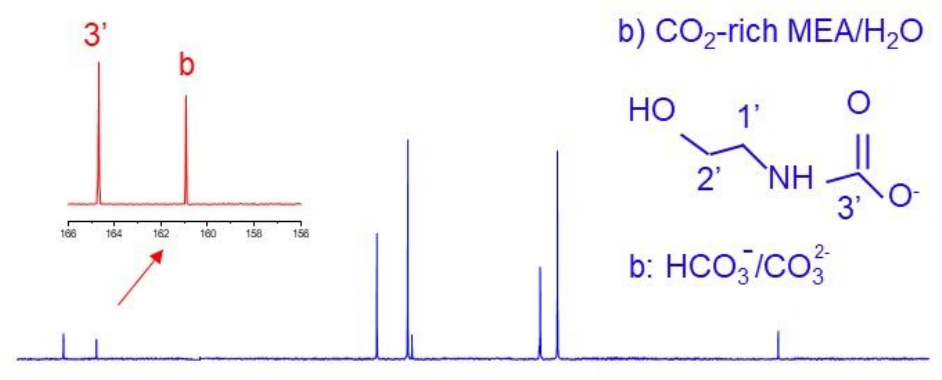

a) Fresh $\mathrm{MEA} / \mathrm{H}_{2} \mathrm{O}$

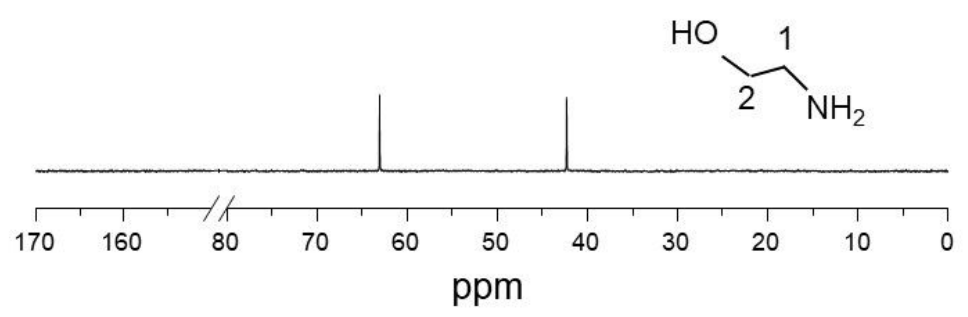

Figure S6. ${ }^{13} \mathrm{C}$ NMR spectra of MEA/ $\mathrm{H}_{2} \mathrm{O}$ absorbents in $\mathrm{D}_{2} \mathrm{O}$. (a) Fresh $\mathrm{MEA} / \mathrm{H}_{2} \mathrm{O}$ absorbent; (b) $\mathrm{CO}_{2}$-rich MEA/ $\mathrm{H}_{2} \mathrm{O}$ absorbent. 
Table S1 Cyclic loading of two absorbents at different MEA concentrations.

\begin{tabular}{|c|c|c|c|c|c|}
\hline Absorbent & $\begin{array}{c}\text { MEA } \\
(\mathrm{mol} / \mathrm{L})\end{array}$ & $\begin{array}{c}1 \mathrm{st} \\
\left(\mathrm{mol}_{\mathrm{CO} 2} / \mathrm{mol}\right. \\
\mathrm{MEA})\end{array}$ & $\begin{array}{c}2^{\text {nd }} \\
\left(\mathrm{mol}_{\mathrm{CO} 2} / \mathrm{mol}\right. \\
\text { MEA })\end{array}$ & $\begin{array}{c}3 \mathrm{rd} \\
\left(\mathrm{mol}_{\mathrm{CO} 2} / \mathrm{mol}\right. \\
\mathrm{MEA})\end{array}$ & $\begin{array}{c}\text { Average } \\
\left(\mathrm{mol}_{\mathrm{CO} 2} / \mathrm{mol}\right. \\
\text { MEA })\end{array}$ \\
\hline \multirow{5}{*}{$\mathrm{MEA} / \mathrm{H}_{2} \mathrm{O}$} & 0.5 & 0.499 & 0.446 & 0.386 & 0.44 \\
\hline & 1 & 0.34 & 0.305 & 0.335 & 0.33 \\
\hline & 2 & 0.294 & 0.249 & 0.252 & 0.27 \\
\hline & 3 & 0.244 & 0.227 & 0.265 & 0.25 \\
\hline & 5 & 0.191 & 0.176 & & 0.18 \\
\hline \multirow{5}{*}{ MEA/PEG200 } & 0.5 & 0.411 & 0.372 & 0.395 & 0.39 \\
\hline & 1 & 0.349 & 0.357 & 0.372 & 0.36 \\
\hline & 2 & 0.357 & 0.364 & 0.357 & 0.36 \\
\hline & 3 & 0.337 & 0.324 & 0.32 & 0.33 \\
\hline & 5 & 0.257 & 0.244 & & 0.25 \\
\hline
\end{tabular}


Table S2. Changes of weight of C20 steel in different immersion time.

\begin{tabular}{ccccc}
\hline Solution & $\begin{array}{c}\text { Immersion Time } \\
\text { (days) }\end{array}$ & $\begin{array}{c}\text { Weight before } \\
\text { immersion }(\mathrm{g})\end{array}$ & $\begin{array}{c}\text { Weight after } \\
\text { immersion }(\mathrm{g})\end{array}$ & $\begin{array}{c}\text { Corrosion rate } \\
(\%)\end{array}$ \\
\hline MEA/PEG200 & 30 & 1.4045 & 1.4018 & $0.19 \%$ \\
& 120 & 1.4537 & 1.4429 & $0.74 \%$ \\
$\mathrm{CO}_{2}$ rich & 400 & 1.5663 & 1.5558 & $0.67 \%$ \\
$\mathrm{MEA} / \mathrm{PEG} 200$ & 30 & 1.2894 & 1.2808 & $0.67 \%$ \\
& 120 & 1.3500 & 1.3405 & $0.70 \%$ \\
$\mathrm{MEA} / \mathrm{H}_{2} \mathrm{O}$ & 400 & 1.5058 & 1.4954 & $0.69 \%$ \\
& 30 & 1.4185 & 1.4077 & $0.76 \%$ \\
& 120 & 1.4461 & 1.3231 & $8.51 \%$ \\
& 400 & 1.4381 & 1.2881 & $10.43 \%$ \\
\hline $\mathrm{CO}_{2}$ rich MEA $/ \mathrm{H}_{2} \mathrm{O}$ & 30 & 1.5517 & 1.532 & $1.27 \%$ \\
& 120 & 1.3342 & 1.1732 & $12.07 \%$ \\
& 400 & 1.3928 & 1.1132 & $20.07 \%$ \\
\hline
\end{tabular}

